\title{
EP-190
}

\section{Can we predict the need of nutritional support following pancreatoduodenectomy?}

\author{
Deeksha KAPOOR* , Azhar PERWAIZ, Amanjeet SINGH, Adarsh CHAUDHARY
}

Departments of GI Surgery and GI Oncology, Minimal Access and Bariatric Surgery, Medanta - The Medicity, Gurgaon, India

Introduction: Poor nutritional status predisposes to complications after pancreatoduodenectomy (PD) and the development of complications adversely affects the nutritional status. The initially prevalent practise of a routine enteral feeding access at the time of surgery has been challenged in favour of a more selective approach. We hypothesised that if need of nutritional support (NS) could be predicted, a tube jejunostomy (TJ) could be added to the surgical procedure.

Methods: A retrospective study of 562 consecutive patients who underwent PD from March 2013 to December 2020 using selective TJ placement. Need of NS was defined as use of TJ beyond POD 4, initiation of parenteral nutrition (PN) and need of nasojejunal (NJ) tube. Preoperative and intraoperative parameters were analysed to identify factors predicting the need of SN.

Results: Of 562 patients, 105 (18.7\%) needed NS. TJ was performed for 46 (8.2\%) patients, PN used in 83 (14.8\%) and NJ placed for 28 (4.9\%). TJ was most useful in patients with psychiatric disorders. PN was most commonly initiated for delayed gastric emptying (DGE) (70/83, 84.3\%). Male sex and a higher operative blood loss were associated with the need of NS. Since PN was most commonly initiated for DGE and patients with major complications almost always need NS, factors predicting DGE and major complications were assessed. Preoperative gastrointestinal bleed and high creatinine levels were associated with DGE. High creatinine and nodal stage were associated with major complications.

Conclusions: TJ can be selectively added in patients with psychiatric disorders, preoperative gastrointestinal bleed, high creatinine and high operative blood loss. 